\title{
Bonding with boron
}

Long ago, a global search for borane superfuels led fortuitously to the discovery of carboranes.

Ken Wade recalls his own undistinguished part in the space race, and notes how carboranes revitalized boron hydride chemistry and modified our ideas of chemical bonding.

n $\mathrm{n}$ the 1950s, during the space race, the communist east and capitalist west built ever bigger and more powerful rockets to threaten to lob nuclear payloads at each other. Progress was marked by how far down test ranges nose cones were recovered, until bleeps emitted by the first Sputnik showed who had won the race into orbit. Down below, engineers continued designing and building better rocket motors, while chemists sought higher-energy fuels. Boron attracted attention for a while because burning its hydrides (boranes) generated more heat per kilogram than burning hydrocarbons. Although the thermally unstable boranes seemed far from ideal as fuels or fuel additives, the USA and USSR invested millions of dollars and roubles, respectively, in borane research. The UK government, not to be outdone, allocated $£ 600$ for similar work. That paid me to work on boranes with Professor Harry Emeleus at Cambridge (the alternative was to be drafted into the army).

Professor Emeleus was a delightful person to work with, kind, and with a dry sense of humour. He provided me with a face shield, asbestos gloves and a soft leather apron, suggesting, with a twinkle, that, in an explosion, the apron might not stop all the glass fragments, but those that got through should be big enough for the surgeons (plural!) to pick out. Thus encouraged, I studied assorted reactions of diborane $\left(\mathrm{B}_{2} \mathrm{H}_{6}\right)$, condensing small samples with potential oxidants in glass vessels and allowing them to react as liquids or gases as they warmed up. Most did so in a controllable manner.

One day I rashly sealed together diborane and a volatile oxidant that I had felt sure would react immediately at low temperature. It didn't, so hours later my reaction vessel, clamped behind a safety screen in the fume cupboard, contained a potentially explosive mixture of the two gases at room temperature. Suddenly, with

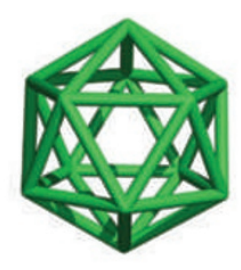

Closo

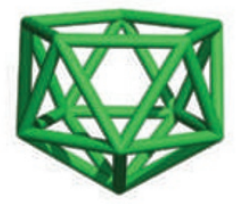

Nido

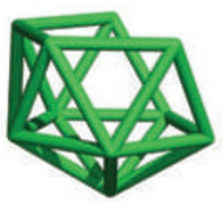

Arachno

Borane-type clusters held together by 13 electron pairs have shapes based on the icosahedron. This is complete (closo) with 12 skeletal atoms, as in $\mathrm{C}_{2} \mathrm{~B}_{10} \mathrm{H}_{12}$ or $\left[\mathrm{B}_{12} \mathrm{H}_{12}\right]^{2-}$ : nest-like (nido, one vacancy) with 11 skeletal atoms, as in $\left[\mathrm{C}_{2} \mathrm{~B}_{9} \mathrm{H}_{11}\right]^{2-}$; and cobweb-like (arachno, two vacancies) with 10 skeletal atoms, as in $\left[\mathrm{B}_{10} \mathrm{H}_{14}\right]^{2-}$.

a greenish flash and a loud bang, the vessel disappeared, most of it so finely pulverized that no one was hurt. The jaws of the clamp that had held it grinned vacantly at me. Only one glass fragment remained. The sealed tube end had shot sideways, creating a bullet hole in the reinforced glass of the fume cupboard, yet falling back intact, like a rocket nose cone awaiting recovery. Was this to be Britain's fuel-oxidant mix? No. Boranes were too expensive to prepare, and proved unsuitable for satellite- or missilelaunching. Other fuel-oxidant systems were developed instead.

However, the money spent globally on boranes was not wasted. It led to the discovery of new borane anions $\left[\mathrm{B}_{n} \mathrm{H}_{n}\right]^{2-}$ and carboranes $\left(\mathrm{C}_{2} \mathrm{~B}_{n-2} \mathrm{H}_{n}\right)$, mixed hydrides of boron and carbon with novel triangularfaced (deltahedral) structures that packed their $n$ boron and carbon atoms closely on spherical surfaces, held together by only $(n+1)$ electron pairs, too few to allocate a pair to each of their $(3 n-6)$ atom-atom links. Dubbed 'electron-deficient', they were really 'electron-sufficient', as adding electrons caused their structures to open up into fragments of bigger deltahedra. Plotting their shapes against formula types or electron numbers creates a pattern of complete deltahedra or fragments thereof, first reported by carborane pioneer Robert E. Williams in 1971, now routinely featured in textbooks. Many other cluster compounds conform to this pattern, as I found by teaching cluster chemistry to Durham students. Boranes, apparent rulebreakers, had become pattern-makers.

Now a mature field, carborane chemistry attracts many talented researchers, in organic, organometallic and inorganic chemistry as well as materials science. It has provided new substituents, ligands, reagents and catalysts, thermally stable polymers, ceramic materials and anticancer drugs. Carborane structures, once hotly disputed, can now be computed with a precision rivalling that of X-ray crystallography. Boranes have featured in the work of such Nobel Laureates as William N. Lipscomb (1976; structures and bonding); Herbert C. Brown (1979; hydroboration studies, so apt for his initials); Roald Hoffmann (1981; structures, bonding and reactions); and indeed George A. Olah (1994; carbocations, analogues of boranes and carboranes). They and many other chemists have been in their element in the fascinating world of boron chemistry.

KEN WADE is Emeritus Professor in the Department of Chemistry at the University of Durham, UK.

e-mail: kenneth.wade@dur.ac.uk 